\begin{tabular}{|c|c|}
\hline Title & Tough double network elastomers reinforced by the amorphous cellul ose network \\
\hline Author(s) & $\begin{array}{l}\text { Murat, Joji; Nakaj ima, Tasuku; Matsuda, Takahiro; Tsunoda, Katsuhiko; Nonoyama, Takay uki; Kurokawa, Takay uki; } \\
\text { Gong, Jian Ping }\end{array}$ \\
\hline Citation & $\begin{array}{l}\text { Polymer, } 178,121686 \\
\text { https://doi.org/10.1016/.polymer.2019.121686 }\end{array}$ \\
\hline Issue Date & 2019-09-12 \\
\hline Doc URL & http:/hdl.handle.net/2115/82657 \\
\hline Rights & $\begin{array}{l}\text { (0) 2019. This manuscript version is made available under the CC-BY-NC-ND } 4.0 \text { license } \\
\text { http://creativecommons.org/icenses/by-nc-nd/4.0/ }\end{array}$ \\
\hline Rights(URL) & https://reativecommons.org/icenses/by-nc-nd/4.0/ \\
\hline Type & article (author version) \\
\hline File Information & Polymer_178_121686.pdf \\
\hline
\end{tabular}

Instructions for use 


\section{Tough Double Network Elastomers Reinforced by the Amorphous Cellulose Network}

Joji Murai ${ }^{1}$, Tasuku Nakajima ${ }^{2,3,4 *}$, Takahiro Matsuda ${ }^{3}$, Katsuhiko Tsunoda ${ }^{5}$, Takayuki

Nonoyama $^{3,4}$, Takayuki Kurokawa ${ }^{3,4}$, Jian Ping Gong ${ }^{2,3,4 *}$

${ }^{1}$ Graduate School of Life Science, Hokkaido University

N10W8, Kita-ku, Sapporo, 060-0810, Japan

${ }^{2}$ WPI-ICReDD, Hokkaido University

N21W10, Kita-ku, Sapporo, 001-0021, Japan

${ }^{3}$ Faculty of Advanced Life Science, Hokkaido University

N21W11, Kita-ku, Sapporo, 001-0021, Japan

${ }^{4}$ Soft-Matter GI-CoRE, Hokkaido University

N21W11, Kita-ku, Sapporo, 001-0021, Japan

${ }^{5}$ Advanced Materials Research Department 1, Central Research, Bridgestone Corporation, Tokyo, 187-8531, Japan

E-mail: tasuku@sci.hokudai.ac.jp,gong@sci.hokudai.ac.jp

Keywords: elastomer; double network; toughening; cellulose; crack growth 


\begin{abstract}
Amorphous cellulose-based tough double-network (DN) elastomers were successfully fabricated. These elastomers comprise interpenetrated poly(ethyl acrylate) (PEA) network as the soft matrix and the amorphous cellulose network as the brittle component. Unlike carbonblack-filled conventional rubbers, the obtained cellulose/PEA DN elastomers are transparent and can be dyed without any color limitation. Although the cellulose network in the DN elastomer comprises only $2.55 \mathrm{wt} \%$, such cellulose network efficiently reinforces in toughness (10 times), stiffness (28 times), strength (8 times), and durability of the DN elastomer compared to the PEA elastomer. The structure and toughening mechanism of the cellulose/PEA DN elastomers are different from previously reported cellulose composites, in which cellulose nanocrystals were used simply as fillers. Upon deformation, the brittle cellulose network in the DN elastomer is ruptured sacrificially to dissipate energy, which effectively prevents crack propagation. The damaged cellulose network recovers its original structure to show recoverable mechanical properties by thermal annealing.
\end{abstract}




\section{Introduction}

Elastomers are unique solid materials with large and reversible deformability. Hence, they are ubiquitous in various industrial products such as tires, sealants, dampers, and sports goods, and are difficult to replace by other materials. For instance, the rubber tread of a tire has been used since the invention of solid rubber tires in the mid-nineteenth century. Such wide applications require elastomers to possess sufficient mechanical robustness. Traditionally, nanofillers such as carbon black (CB) have been added for more than 100 years to increase the toughness and stiffness of the elastomers [1]. However, conventional filled elastomers have raised concerns, mainly because of their undesirable environmental impact. Firstly, CB is generally manufactured from fossil hydrocarbons, which are facing depletion. Secondly, the CB microparticles used as fillers cause air and water pollution if removed from the elastomer. Thirdly, massive amounts of filler particles (typically 10-50 vol\%) are required for an effective reinforcement of the elastomers [1]. Furthermore, the color of the filled elastomer is limited by the color of the filler, which restricts the range of applications; for example, the use of CB-filled rubber has been avoided in some warehouses and hospitals to prevent formation of black stains. Recently, instead of the CB fillers, silica fillers are also used. Comparing with the $\mathrm{CB}$ filler, one large drawback is the increased weight density, which is more energy consumptive. To overcome the above-mentioned environmental concerns caused by the use of the fillers, a novel eco-friendly method for toughening of the elastomers is required.

Recently, several studies on novel toughening methods of gels, which are rubbery materials containing solvent, have been reported. Examples include slide-ring systems [2,3], polymer-clay nanocomposites [4,5], and the sacrificial bond (s-bond) principle [6,7]. Among these, we have focused on an approach based on the s-bond principle. A toughened gel following the s-bond principle typically consists of a highly stretchable matrix with dense brittle bonds (s-bonds). The s-bond principle was originally applied for preparing doublenetwork (DN) gels comprising a covalently cross-linked brittle first network and a stretchable 
second network $[6,8]$. If a force is applied to a DN gel, the chemical bonds of the brittle first network (i.e., s-bonds) break sacrificially to dissipate large energy while the stretchable second network maintains the integrity of the DN gels $[9,10]$. This energy dissipation mechanism sufficiently retards crack propagation at the crack tip of the DN gels and furnishes extremely high fracture toughness [11-14]. Various chemical bonds such as covalent, ionic, and hydrogen $(\mathrm{H})$ bonds can be used as s-bonds for toughening of gels $[6,7,15-19]$. If irreversible covalent bonds are used as s-bonds, then the broken sacrificial bonds cannot be reformed. Therefore, the toughness of such s-bond gels (such as DN gels) decreases once they are damaged. However, if non-covalent interactions are used as s-bonds, the gels can recover from the accumulated damage because of the reversible nature of non-covalent s-bonds.

Since both ideal gels and elastomers are soft materials that display rubber-like elasticity, the s-bond principle should also be applicable for elastomer toughening. An early example of an s-bond elastomer was a triple network (TN) elastomer, consisting of a covalently crosslinked first network with brittle s-bonds and a stretchable third network as the soft matrix (the role of the second network was to make the first network brittle) [20,21]. The reported TN elastomer showed a high Young's modulus (4 MPa) as well as a high fracture energy $\left(9 \mathrm{~kJ} \mathrm{~m}^{-}\right.$ ${ }^{2}$ ) due to the brittle first network working as s-bonds [20]. Although the TN elastomer concept has proven that the s-bond principle can be applied to elastomer toughening, TN elastomers have irreversible covalent bonds in the first network as s-bonds, implying that there would be no recoverability of the mechanical properties once the elastomer is damaged.

To overcome the environmental problems of CB-filled rubbers as well as the recoverability problem of TN elastomers, here we report the novel and eco-friendly cellulose/poly(ethyl acrylate) (PEA) DN elastomers with damage recoverability based on the s-bond principle. This cellulose/PEA DN elastomer was composed of a brittle cellulose network (providing s-bonds) and the PEA elastomer, also known as acrylic rubber, serving as the soft matrix. Cellulose is the most common and eco-friendly organic compounds produced 
by plants and some bacteria. Although various cellulose-reinforced polymeric materials have already been reported [22-29], the concept and the toughening mechanism of our DN elastomer are completely different from the reported ones. Many researchers have used discontinuous cellulose nanocrystals as nanofillers to toughen elastomers [22,25-27]. However, effective stiffening and toughening of the elastomer usually requires a relatively large amount and well-controlled dispersion of cellulose nanocrystals. Bacterial cellulose (BC) gels having a continuous network have been also used as the first network of the DN gels $[28,29]$. However, BC gels consisting of thick cellulose bundles are too strong to function as s-bonds. Alternatively, in this study, the amorphous cellulose network prepared from molecularly-dispersed cellulose has been adopted as the brittle first network that provides sbonds to effectively toughen the DN elastomer.

\section{Experimental}

Materials: Filter paper ADVANTEC No.1 (Toyo Roshi Kaisha, Ltd.) was used as the cellulose source. Lithium chloride anhydrous ( $\mathrm{LiCl}), N, N$-dimethylacetamide (DMAc), 4dimethylaminopyridine (DMAP), ethyl acrylate (EA), and ethylene glycol dimethacrylate (EGDMA) were purchased from Tokyo Chemical Industry Co., Ltd. 1,2,3,4Butanetetracarboxylic dianhydride (BTCA) and acetone were purchased from Wako Pure Chemical Industries, Ltd. Benzophenone was purchased from Kanto Chemical Co., Inc. Ethanol was purchased from Japan Alcohol Corporation Inc.. All the chemicals were used without any further purification.

Preparation of the cellulose gel: The filter paper was cut into small pieces and immersed for 1 day each in excess pure water, acetone, and DMAc. Then, the filter paper pieces were dried in vacuo at $60{ }^{\circ} \mathrm{C}$ for 1 day to remove residual solvents. Subsequently, $8 \mathrm{wt} \% \mathrm{LiCl}$ was added to DMAc; the solution was stirred at $100{ }^{\circ} \mathrm{C}$ for $3 \mathrm{~h}$ and then at room temperature for $2 \mathrm{~h}$ to 
obtain $8 \mathrm{wt} \% \mathrm{LiCl} / \mathrm{DMAc}$ solution. $0.5 \mathrm{~g}$ of the washed filter paper was dissolved in $49.5 \mathrm{~g}$ of the $\mathrm{LiCl} / \mathrm{DMAc}$ solution by stirring for 2 days at room temperature to obtain a transparent 1 $\mathrm{wt} \%$ cellulose solution. BTCA (cross-linker), DMAP (catalyst for the cross-linking reaction), and a very small amount of water were added to this $50 \mathrm{~g}$ cellulose solution. Unless mentioned specifically, the added amounts of BTCA, DMAP, and water were fixed at $0.46 \mathrm{~g}$, $1 \mathrm{~g}$, and $500 \mu \mathrm{L}$, respectively. The solution was poured between a pair of glass plates with 2 mm spacing and kept for 1 day at room temperature. The obtained cellulose gel was then immersed in EtOH for at least 1 day until it reached equilibrium.

Synthesis of the cellulose/PEA DN elastomer: The cellulose organogel as the first network (2 $\mathrm{mm}$ in thickness) was immersed in the excess ethyl acrylate (EA) mixture containing 0.2 mol\% ethylene glycol dimethacrylate (EGDMA; cross-linker) and $1.25 \mathrm{wt} \%$ of benzophenone (initiator) for 1 day until equilibrium was reached. Note that no solvent is used at this step. The gel was then sandwiched between two soda-lime glass plates with a thickness of $3 \mathrm{~mm}$, and polymerization was carried out in the presence of the first cellulose network under irradiation by $365 \mathrm{~nm}$ UV light with an intensity of $4 \mathrm{~mW} / \mathrm{cm}^{2}$, for $8 \mathrm{~h}$ in an argon atmosphere.

Uniaxial stretching measurements: Uniaxial tensile tests, tensile stress relaxation tests, and cycle tensile tests were performed on dumbbell-shaped specimens standardized as JIS K62517 (12 mm length and $2 \mathrm{~mm}$ width). The PEA and DN elastomers were measured as they were, while the cellulose gel was immersed in the EA mixture used for the second network preparation before measurement to mimic the cellulose network condition in the DN elastomer. The tests shown in Figure 3 were performed with an Instron 5965 tensile tester (Instron Co.), while the other tests were performed with an Autograph AG-X plus (Shimadzu Co.) equipped with a thermo-hygrostat TCL (Shimadzu Co.). For uniaxial tensile tests, the 
bottom end of the specimen was fixed and the upper end was pulled up at constant velocity. Tensile velocity and testing temperature were $100 \mathrm{~mm} / \mathrm{min}$, which gives a strain rate $0.14 \mathrm{~s}^{-1}$, and $25^{\circ} \mathrm{C}$, respectively, unless specially mentioned. The tensile fracture stress, $\sigma_{\mathrm{f}}$, and fracture strain, $\varepsilon$, were determined as the nominal stress, $\sigma$, and nominal strain, $\varepsilon$, at the breaking point, respectively. Young's modulus, $E$, was defined as the slope of the stress-strain curves over the strain range $0<\varepsilon<0.1$. For the cycle tensile tests, the specimen was subjected to continuous uniaxial loading and unloading process with constant tensile velocity of $100 \mathrm{~mm} / \mathrm{min}$. For the stress relaxation tests, uniaxial strain of 1 is initially applied to the specimen with loading velocity of $100 \mathrm{~mm} / \mathrm{min}$, and the specimen was allowed to relax for $6,000 \mathrm{~min}$. Time origin $(t=0)$ is defined as the point when the strain reaches to 1 . Stress ratio is defined as $\sigma / \sigma 0$, where $\sigma_{0}$ is the stress at $t=0$.

Tearing tests: Tearing tests were performed on the trouser-shaped specimens standardized as per the JIS-K6252 at $25{ }^{\circ} \mathrm{C}$ with an Instron 5965 tensile tester. The tearing velocity at a crack tip was approximately $50 \mathrm{~mm} / \mathrm{min}$. The tearing energy $T$ is defined as the work required for making a unit area of fracture surface by tearing, as estimated by $T=2 F_{\text {ave }} / h$, where $F_{\text {ave }}$ is the average force during steady tearing, and $h$ is the thickness of the specimen. Note that the stretch of the legs of the specimens is assumed to be negligible.

Crack growth measurements: Crack growth measurements were performed on the rectangular-shaped (so-called "pure shear") specimens (120 mm in width $(x), 20 \mathrm{~mm}$ in height (y), and $\sim 1.5 \mathrm{~mm}$ in thickness) with an electromagnetic testing machine EMT5kN (Shimadzu Co.) at $24^{\circ} \mathrm{C}$. The sample geometry and the experimental procedure are shown in Figure S1. The specimens were uniaxially stretched along the $y$ direction with a constant speed of 1 $\mathrm{mm} / \mathrm{s}$ to the desired strain $\varepsilon$. The input energy density $W$ is defined as $W(\varepsilon)=\int_{0}^{\varepsilon} \sigma(\varepsilon) d \varepsilon$. 
Immediately after imposing the desired strain, a cut was made at the edge of the specimen along $x$ direction and then the crack propagates spontaneously. The crack growth was recorded by a high-speed camera Photron FASTCAM SA4, and the crack velocity $v(\varepsilon)$ was evaluated as the velocity of the steady crack growth based on the taken pictures. Fracture energy $\Gamma$ at each imposed strain was calculated by $\Gamma(\varepsilon)=W(\varepsilon) l_{0}$, where $l_{0}$ is the initial gauge length of the specimen. Note that the input energy density $W$ calculated from the loading curves is not purely elastic energy but also contains dissipateable component.

Spectroscopic characterization. Attenuated total reflectance Fourier transform infrared (ATRFTIR) spectra were recorded with an IR microscope ATR-IR IRT-3000N (JASCO Co.) equipped with a zinc selenide prism. The measured wavenumber range was 500 to $4000 \mathrm{~cm}^{-1}$. Wide-angle X-ray diffraction (WAXD) measurements were performed at BL40B2, SPring-8, Japan, using a complementary metal oxide semiconductor flat panel detector. The X-ray wavelength was $1 \AA$, and the camera length between the sample and the detector was 75.261 $\mathrm{mm}$. 


\section{Results}

\section{Structure Evaluation of the Cellulose/PEA DN elastomer}

A schematic representation of sample preparation is given in Figure 1(a). Filter papers (crystalline cellulose) were dissolved in LiCl/DMAc solutions to obtain a molecular-level cellulose dispersion [30,31]. Then, the chemical cross-linking reagent BTCA and a small amount of water were added to the cellulose solutions. BTCA induced the cross-linking reaction and water induced physical aggregation (H-bonding) between the neighboring cellulose chains [32]. The obtained cellulose gel was highly transparent (Figure 1(b)). Finally, the stretchable PEA network was synthesized in the presence of the cellulose network to obtain the cellulose/PEA DN elastomers. The weight fraction of the cellulose network in the typical cellulose/PEA DN elastomer was only $2.55 \mathrm{wt} \%$. Figure $1(\mathrm{c})$ shows the appearance of the DN elastomer and the conventional CB-filled rubber. The DN elastomer containing both the cellulose and the PEA networks maintained its transparency. Thus, unlike conventional CB-filled black rubber, the DN elastomer can be colored using various pigments.

The transmission electron microscopy (TEM) images of the cellulose/PEA DN elastomer are shown in Figure 2(a). Although structures with a diameter of $\sim 10 \mathrm{~nm}$, which may correspond to the cellulose aggregation, could be identified in the images (at the damaged part), their size was much smaller than the wavelength of visible light. Such well-dispersed cellulose resulted in high transmittance of the cellulose gels and DN elastomers. The wideangle X-ray diffraction (WAXD) spectra are shown in Figure 2(b). Unlike the crystalline cellulose showing some sharp diffraction peaks [23], no crystalline peaks but only a halo peak was found in the spectrum of the cellulose gel, suggesting that the re-formed cellulose network remains in the amorphous state without re-crystallization. The halo peak of PEA also indicates its amorphous state. Figure 2(c) shows that the differential WAXD spectrum between the DN elastomer and PEA elastomer is quite similar to the spectrum of the cellulose gel. It means that the spectrum of the DN elastomer was the sum of the spectra of cellulose 
and PEA, suggesting that the nanoscale structure of the DN elastomer is also simply the sum of the structures of the two components. Figure 2(d) shows the Fourier transform infrared (FT-IR) spectra of the DN elastomer and its counterparts. The broad peak around 3,300 $\mathrm{cm}^{-1}$, which corresponds to $\mathrm{O}-\mathrm{H}$ stretching modes of the $\mathrm{H}$-bonded cellulose chains, was found in the spectrum of the cellulose gel [33]. The other peaks of the cellulose gel in the fingerprint regime are not clearly found because of low concentration of the cellulose. This broad peak also appeared in the spectrum of the DN elastomer, suggesting the existence of inter- or intranetwork $\mathrm{H}$-bonding in that. If the cellulose forms strong inter-network H-bonds with the PEA network in the DN elastomer, such H-bonds should lead peak shift of the PEA network spectrum. However, any significant peak shift is not found between the spectra of the PEA and the DN elastomers. In short summary, no evidence of the intra-network H-bonds is found from the FT-IR spectra, and the cellulose may form the intra-network H-bonds in the DN elastomer. These structural analyses suggested that there was negligible chemical association between the first and the second networks at the molecular level. The two networks of the DN elastomer interact mainly by topological entanglements.

(a)

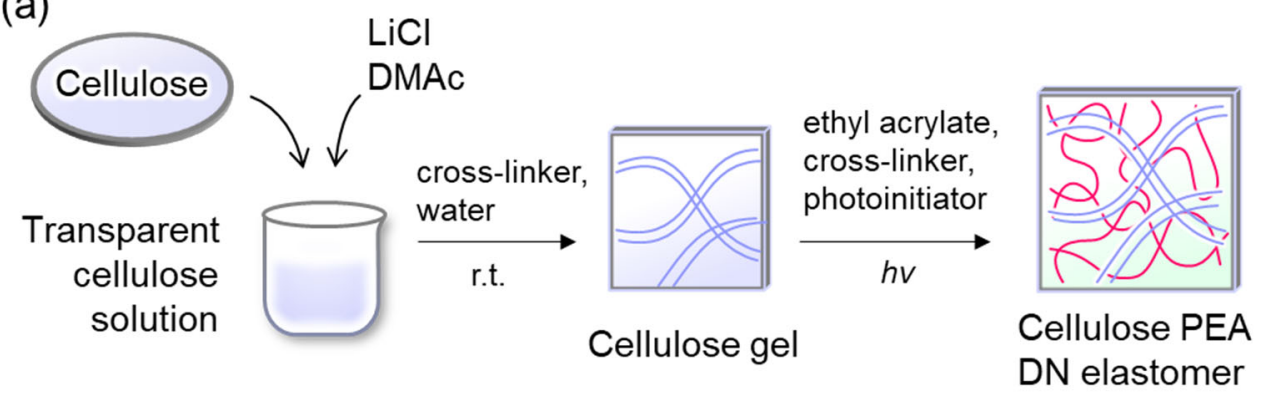

(b) Cellulose gel

(c) Cellulose/PEA DN elastomer dyed by various pigments

CB-filled rubber
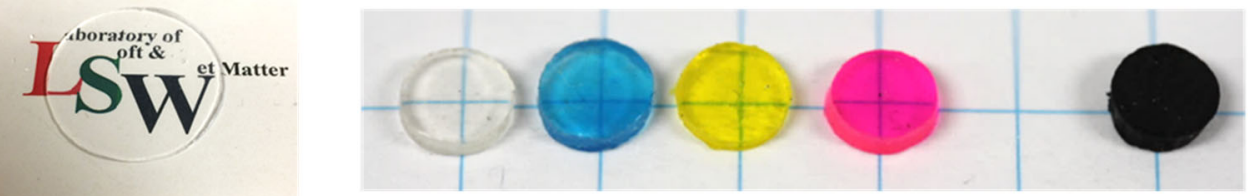
Figure 1. Preparation of the cellulose/PEA DN elastomer. (a) Schematic representation of the synthesis of cellulose/PEA double network elastomers. (b) Appearance of the cellulose gel. (c) Appearance of the cellulose/PEA elastomer dyed with several pigments and the carbon black-filled styrene-butadiene rubber.

(a)

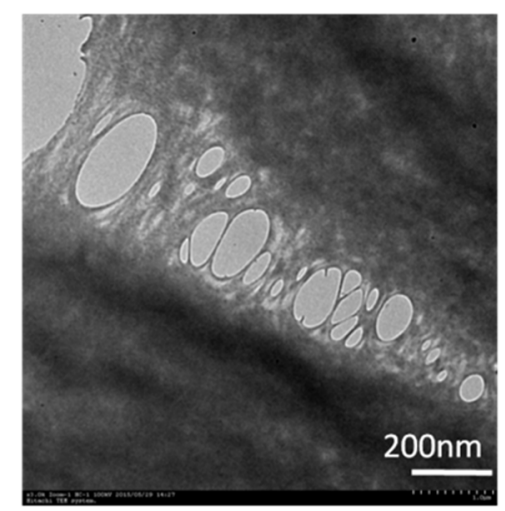

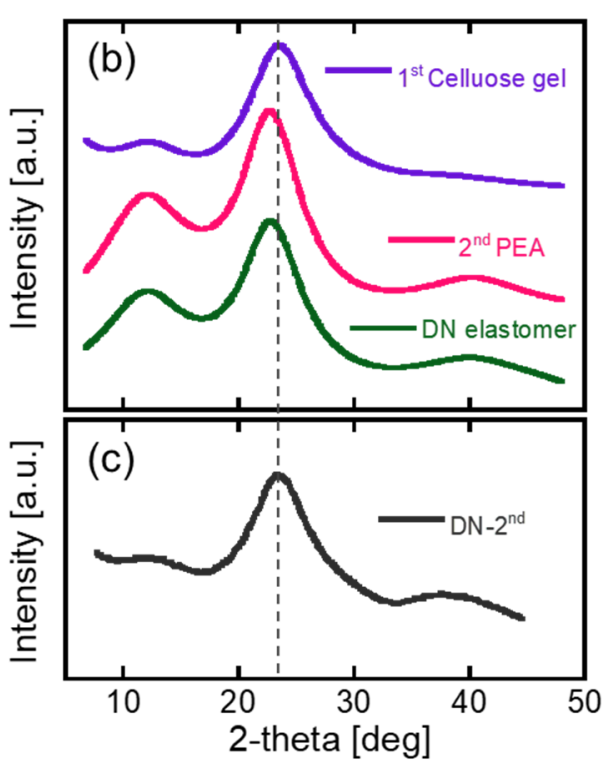

(d)

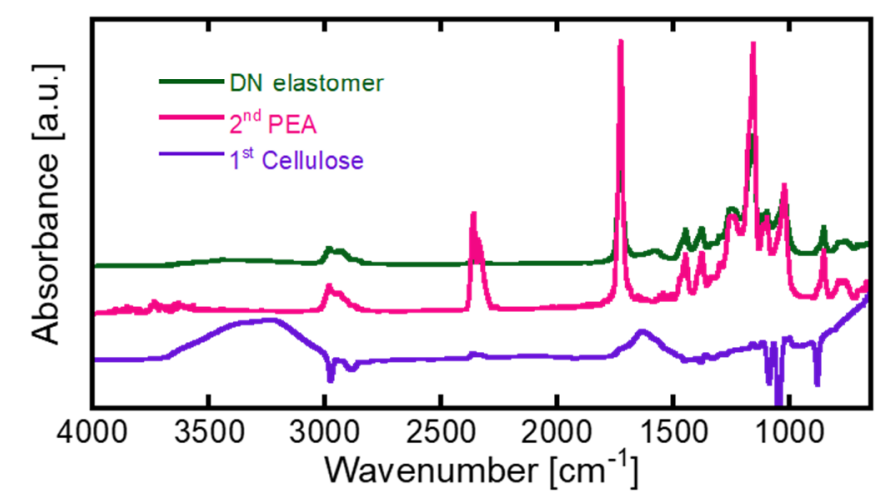

Figure 2. Structure evaluation. (a) A transmittance electron microscope image of the cellulose/PEA DN elastomer. The sample got damaged because of the strong electron beam. (b) Wide-angle X-ray diffraction (WAXD) spectra of the cellulose gel, PEA elastomer, and DN elastomer. The results are offset for clarity. (c) Differential WAXD spectrum between the DN elastomer and the cellulose gel. (d) Fourier transform infrared (FT-IR) spectra of the cellulose gel, the PEA elastomer, and the DN elastomer. The results are offset for clarity. 


\section{Tensile test results of the cellulose/PEA DN elastomer}

Figure 3(a) shows the stress-strain curves of the cellulose gel, PEA elastomer, and a typical DN elastomer. The cellulose gel was stiff but very brittle (Young's modulus $E=9.50$ $\mathrm{MPa}$, tensile fracture stress $\sigma_{\mathrm{f}}=1.27 \mathrm{MPa}$, and tensile fracture strain $\varepsilon_{\mathrm{f}}=0.36$ ), whereas the PEA elastomer was soft and stretchable $\left(E=0.33 \mathrm{MPa}, \sigma_{\mathrm{f}}=0.86 \mathrm{MPa}\right.$, and $\left.\varepsilon \mathrm{f}=8.16\right)$. The cellulose/PEA DN elastomer, consisting of both the cellulose and PEA networks, possessed excellent mechanical properties. The DN elastomer showed a large value of $E(9.19 \mathrm{MPa})$, which almost followed a general mixing rule for composites, whereby the resulting properties follow the sum of the components because of the molecular level independency of the two networks. On the other hand, the DN elastomer had a large tensile strength $\left(\sigma_{\mathrm{f}}=6.51 \mathrm{MPa}\right)$, which greatly exceeded the sum of the values of each network. Despite its large stiffness and strength, the DN elastomer also showed large stretchability $\left(\varepsilon_{\mathrm{f}}=6.30\right)$. It means the cellulose/PEA DN elastomer is a rare example of elastomers which show both high modulus and stretchability, which are typically in trade-off relationship. The high stretchability of the DN elastomer was accompanied with a yielding-like behavior, which is generally observed in conventional DN gels showing high toughness.

\section{Fracture test results of the cellulose/PEA DN elastomer}

Fracture tests of the cellulose/PEA DN elastomer and the PEA elastomer were performed to evaluate their toughness and durability. Note that the cellulose gel was too brittle to be subjected to these tests. Figure 3(b) shows the tearing test results of the trousershaped DN elastomer and the PEA elastomer. The DN elastomer showed high resistance against crack propagation. The measured tearing energy $T$ of the DN elastomer $\left(18,838 \mathrm{~J} \mathrm{~m}^{-2}\right)$ was greatly enhanced over that of the second network (PEA, $T=1,820 \mathrm{~J} \mathrm{~m}^{-2}$ ).

Figure 3(c) shows the pure-shear crack growth test results of the DN and the PEA elastomers. The graph shows the relationship between the crack velocity $v$ and the fracture 
energy $\Gamma$, measured at various input strain $\varepsilon$. The two power-law relationships are found; one is called "slow mode" with low $v$ and $\Gamma$, and the other is called "fast mode" with large $v$ and $\Gamma$. The discontinuous jump of the crack velocity can be found between these two regimes, which has been observed by some previous crack growth experiments for various rubbers and by the finite element method simulation [34-37]. The origin of the jump is attributed to viscoelastic-elastic transition at the crack tip [38]. Empirically, it is known that the threshold $\Gamma$ for the jump (mode transition), $\Gamma_{\text {trans, }}$ is related to durability of the elastomeric materials. If the mode transition occurs at higher $\Gamma_{\text {trans, }}$, such elastomers have the higher durability and longer lifetime and vice versa. In this study, the $\Gamma_{\text {trans }}$ of the cellulose/PEA DN elastomer was measured as $69,300 \mathrm{~J} \mathrm{~m}^{-2}$, whereas that of the PEA elastomer was $2,170 \mathrm{~J} \mathrm{~m}^{-2}$, suggesting the cellulose/PEA DN elastomer is much more durable than the PEA elastomer. In addition to the difference in $\Gamma_{\text {trans, }}$, the slopes of the fast and slow modes of the DN elastomer is much larger than those of the PEA elastomer. The theoretical work about the velocity jump suggests that the slopes are determined by several material constants such as the modulus ratio of the elastomers between their glassy state and the rubbery state [38]. The relationship between the special network structure of the cellulose/PEA DN elastomer and these material constants is the remaining question.

Here, we emphasize that the strength, toughness and durability of the DN elastomer are considerably improved by the addition of only $2.55 \mathrm{wt} \%$ of cellulose, while typical carbon black-filled elastomers contain $10-50 \mathrm{wt} \%$ of CB. In order to compare the toughening efficiency of the cellulose network and $\mathrm{CB}$, the toughening ratio of the elastomers is defined as $T_{\mathrm{r}} / T_{0}$, where $T_{\mathrm{r}}$ and $T_{0}$ correspond to the $T$ of the cellulose/filler-reinforced and nonreinforced elastomers, respectively. The toughening ratio of the $2.55 \mathrm{wt} \%$ cellulose network in PEA was 10.4, which greatly exceeded that of the $30 \mathrm{wt} \% \mathrm{CB}$ for vulcanized natural rubber (NR) and styrene-butadiene rubber (SBR) (3.3 and 3.9, respectively [1]). This extraordinary 
improvement of the properties of the DN elastomer strongly suggests a synergistic toughening effect of the two interpenetrated networks in the elastomer, although the networks have negligible interaction at the molecular level.
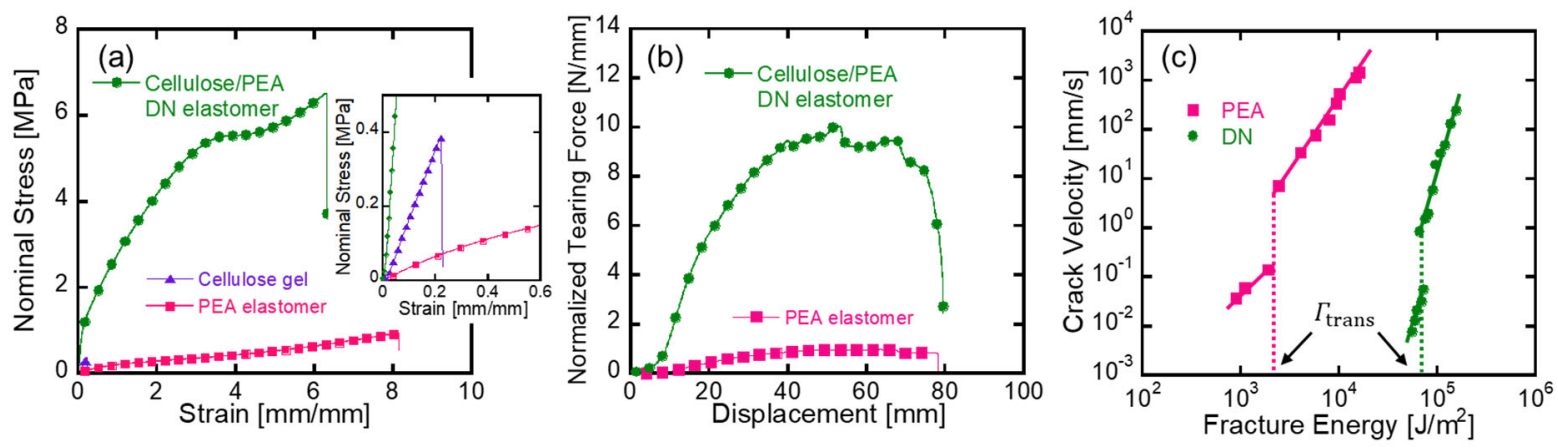

Figure 3. Mechanical properties. (a) Uniaxial tensile stress-strain curves of the cellulose gel, PEA elastomer, and cellulose/PEA DN elastomer. (b) Normalized tearing force-displacement curves of the trouser-shaped PEA and DN elastomers. (c) The relationship between the crack velocity $v$ and the fracture energy $\Gamma$ of the PEA and DN elastomers measured by the crack growth test. The solid lines represent the two power-law relationships which correspond to the slow and the fast fracture modes, and the dotted line represents the fracture energy at the mode transition, $\Gamma$ trans.

\section{Effect of the Cross-linking Condition of the Cellulose Network}

The abovementioned excellent mechanical properties of the cellulose/PEA DN elastomer are strongly affected by the cross-linking conditions of the cellulose first network. Figure 4(a) shows the effect of the amount of chemical cross-linker BTCA used in the preparation of the first cellulose network on the results of the tensile test. The DN elastomer without BTCA was quite strong but brittle. With an increase in the amount of BTCA, the strength of the DN elastomers decreased but their extensibility increased, and $0.46 \mathrm{~g}$ of BTCA added to $50 \mathrm{~g}$ of the cellulose solution resulted in the best DN elastomer with both high strength and extensibility. This dependence of the mechanical properties on the BTCA concentration might be a result of both the chemical modification and the structure fixing of the cellulose chains. When cellulose chains dissolved in $\mathrm{LiCl} / \mathrm{DMAc}$ are re-aggregated, the inter-chain $\mathrm{H}$-bonds 
are formed again, leading to physical gelation of the cellulose. At this time, the chemical modification of cellulose by BTCA decreases the density of the $\mathrm{OH}$ groups and thus chemically disturbs the inter-chain H-bond formation. Moreover, the chemical cross-linking of the cellulose by BTCA restricts the motion and re-aggregation of the cellulose chains, which also disturb the H-bond formation topologically. Therefore, it is expected that an increase in the BTCA concentration would decrease the number of $\mathrm{H}$-bonds, which in turn would decrease the strength of the cellulose network. According to a previous study, weakening of the first brittle network leads to a decrease in the strength of the DN system but an increase in its extensibility $[39,40]$. The dependence of the mechanical properties of the DN elastomers on the BTCA concentration can be understood based on a similar rationale. As the re-aggregation and gelation of cellulose is directly influenced by the presence of water, the properties of the DN elastomers are also strongly influenced by the amount of water used in the preparation of the cellulose gel. As shown in Figure 4(b), the tensile properties of the cellulose/PEA DN elastomers were strongly influenced even by slight changes in the water concentration from 0 to $20 \mu \mathrm{L} \mathrm{g}^{-1}$, and the concentration of $10 \mu \mathrm{L} \mathrm{g}^{-1}$ gave the best strength, stiffness, and extensibility of the DN elastomers. In the absence of water, the lack of H-bonds rendered the obtained cellulose gel too weak for the first network of the DN elastomers. The presence of $10 \mu \mathrm{L} \mathrm{g}^{-1}$ water resulted in the most suitable interactions for the cellulose network, rendering a sufficiently tough DN elastomer. 

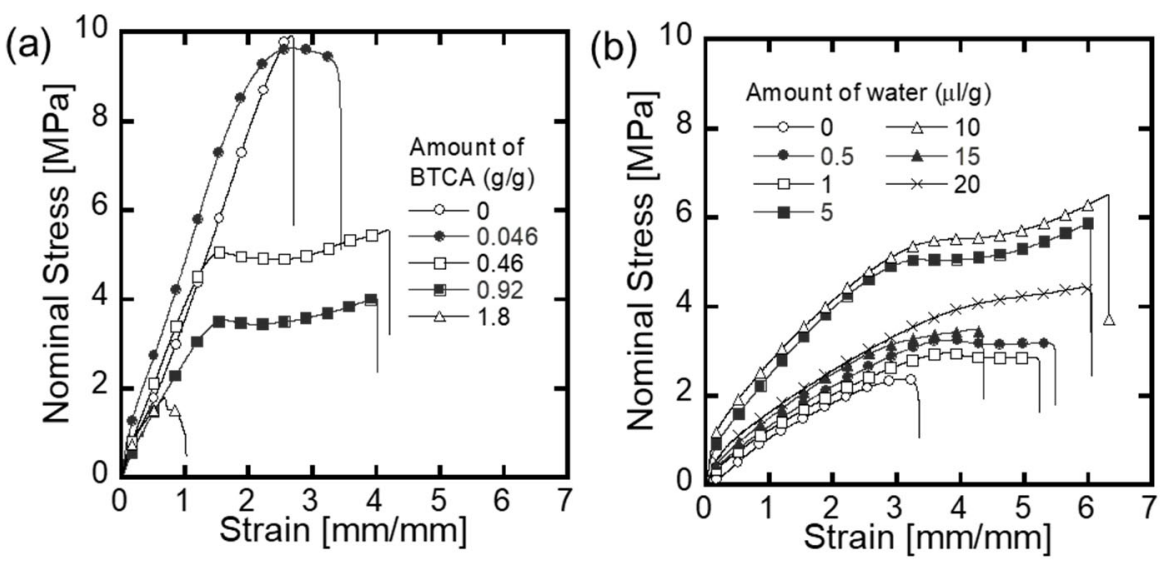

Figure 4. Effect of the cross-linking conditions. (a) Effect of the amount of BTCA added to the cellulose solution on the stress-strain curves of the cellulose/PEA DN elastomers. (b) Effect of the amount of water added to the cellulose solution on the stress-strain curves of the DN elastomers.

\section{Relaxation and Damage Recovery}

To analyze the origin of the synergistic toughening of the DN elastomer, the tensile stress relaxation tests (applied strain $\varepsilon=1$ ) were performed. Figure 5(a, b) shows the stress ratio, $\sigma / \sigma$, versus relaxation time, $t$, curves of the PEA elastomer and the DN elastomer. Relaxation behavior of the PEA elastomer can be fitted with the stretched single-exponential model having one relaxation time constant, $\tau$, as

$$
\frac{\sigma}{\sigma_{0}}=\sigma_{\infty}+\sigma_{1} \exp \left(-\left(\frac{t}{\tau}\right)^{\beta}\right)
$$

where $\sigma_{\infty}$ is the equilibrium stress ratio and $\beta$ is the stretching exponent. The obtained fitting parameters were $\sigma_{\infty}=0.66, \sigma_{1}=0.34, \tau=1.1 \times 10^{2} \mathrm{~s}$, and $\beta=0.41$. This relatively slow relaxation of $\tau \sim 100 \mathrm{~s}$ may correspond to the longest Rouse mode of the PEA chains. In contrast, the DN elastomer showed two-step (fast and slow) relaxation behavior. This relaxation curve was well fitted by the stretched double-exponential relaxation equation having two time constants $\tau_{1}$ and $\tau_{2}\left(\tau_{1}<\tau_{2}\right)$ as

$$
\frac{\sigma}{\sigma_{0}}=\sigma_{\infty}+\sigma_{1} \exp \left(-\left(\frac{t}{\tau_{1}}\right)^{\beta_{1}}\right)+\sigma_{2} \exp \left(-\left(\frac{t}{\tau_{2}}\right)^{\beta_{2}}\right)
$$


with the fitting parameters of $\sigma_{\infty}=0.38, \sigma_{1}=0.43, \tau_{1}=2.0 \mathrm{~s}, \beta_{1}=0.41, \sigma_{2}=0.18, \tau_{2}=$ $1.0 \times 10^{3} \mathrm{~s}$, and $\beta_{2}=0.70$. These fast and slow relaxations should originate from either relaxation of the PEA chains or that of the cellulose chains. The slow relaxation of $\tau_{2} \sim 1,000$ $\mathrm{s}$ is probably attributed to the Rouse mode of PEA chains trapped in a higher fractional tube constituted by the first network. The slower $\tau_{2}$ of the DN elastomer than $\tau$ of the PEA elastomer is possibly caused by the topological confinement of the PEA network in the DN elastomer via interpenetrating network structure. Meanwhile, the fast relaxation of $\tau_{1} \sim 1 \mathrm{~s}$ possibly originates from the association (H-bonding) between the cellulose chains, since such very fast relaxation was not found in the PEA elastomer.

Effect of this fast relaxation of the DN elastomer to its mechanical behavior was investigated by continuous loading-unloading tensile tests at an initial strain rate of $0.14 \mathrm{~s}^{-1}$, which is slow enough for the fast relaxation and fast enough for the slow relaxation mode (Figure 5(c, d)). The PEA elastomer had a negligible hysteresis loss up to a strain of 3, suggesting that the PEA elastomer is almost elastic at such a short time scale. In contrast, the DN elastomer showed obvious mechanical hysteresis loss. It is possibly caused by internal rupture of H-bonding of the cellulose network having small $\tau$, which induces energy dissipation. This hysteresis loss was recovered partially in the next cycle, suggesting a part of the ruptured bonds re-formed during the unloading process.

This damage recovery behavior of the DN elastomer should depend on the temperature. To examine it, the loading-unloading test was performed for four times on the DN elastomer with the maximum strain $\varepsilon_{\max }=1$. Between each cycle, the samples were annealed at the desired temperature for $5 \mathrm{~min}$ and then cooled at $25{ }^{\circ} \mathrm{C}$ for $5 \mathrm{~min}$ to promote the re-formation of the H-bonds. The recovery ratio was calculated as $W_{2-4} / W_{1}$, where $W_{2-4}$ is the average work of extension at $\varepsilon_{\max }=1$ of the $2^{\text {nd }}-4^{\text {th }}$ loading, and $W_{1}$ is the work of the $1^{\text {st }}$ loading. The $W_{2}$ $4 / W_{1}$ ratio increased with the temperature and finally reached around $90 \%$ at over $100{ }^{\circ} \mathrm{C}$, as 
shown in Figure 6(a). We also examined the maximum strain dependence of the recovering property, as shown in Figure 6(b). The annealing temperature was chosen as $130{ }^{\circ} \mathrm{C}$. At $\varepsilon_{\max }$ $<1$, the DN elastomer recovered over $90 \%$ of the original work of extension, whereas at $\varepsilon_{\max }$ $>1$, it showed incomplete self-recovery even after annealing. These results suggest the occurrence of a two-step internal fracture of the cellulose network in the DN elastomers. At $\varepsilon$ $<1$, the energy loss of the DN elastomers was completely recoverable by thermal annealing, indicating reversible structure change occurred in this region. On the other hand, at $\varepsilon>1$, the DN elastomer had undergone irreversible structure change. It should be noted that the $2^{\text {nd }}$ and $3^{\text {rd }}$ loading curves of the DN elastomer showed higher stress than the $1^{\text {st }}$ loading curve at the strain of 1. This implies that the network structure of the as-prepared DN elastomer is metastable, which leads lower stress of the $1^{\text {st }}$ loading curve. The network structure then transits to its more stable states with the thermal treatment, which may lead higher stress of the $2^{\text {nd }}$ and $3^{\text {rd }}$ loading curves. 

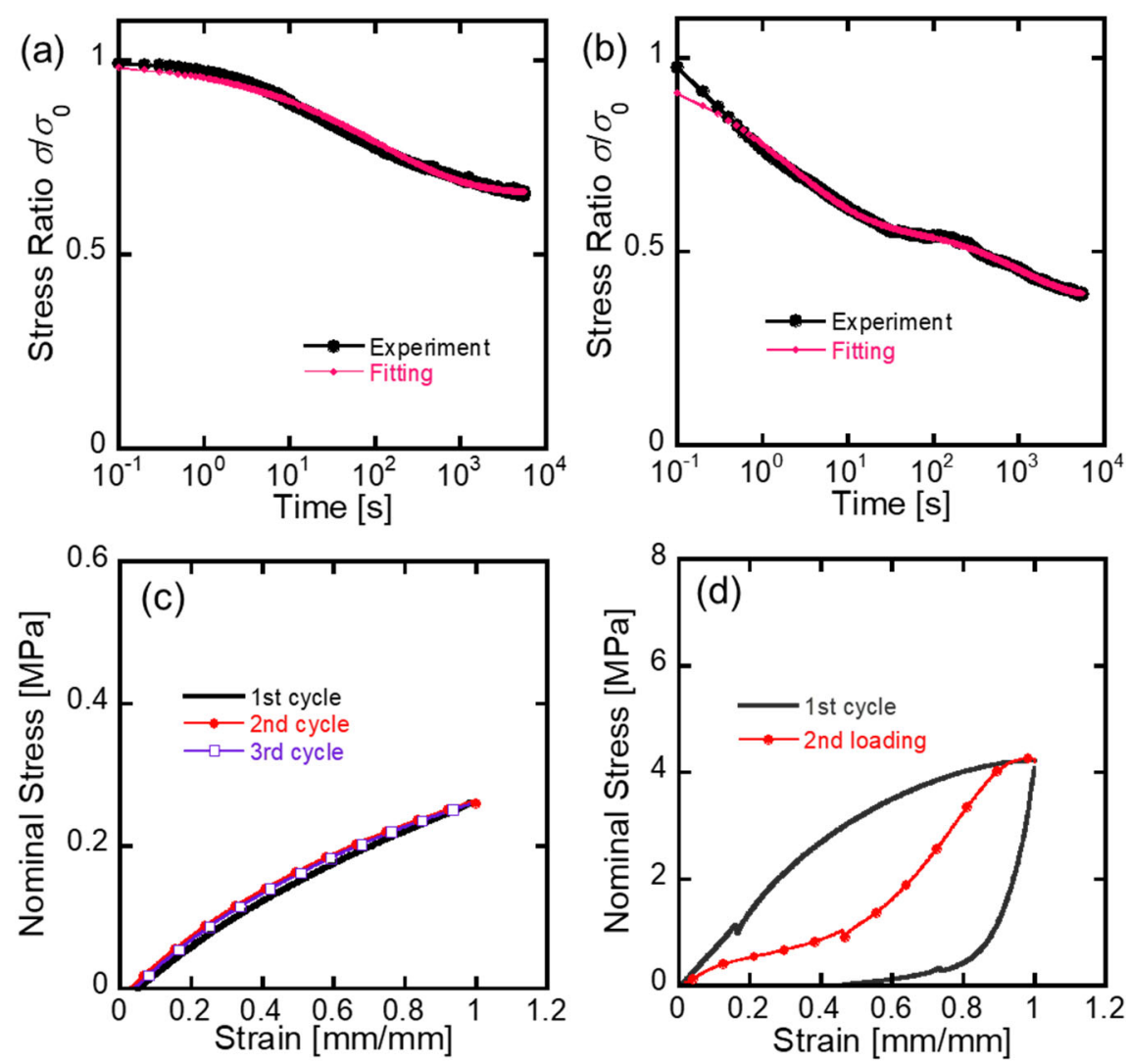

Figure 5. Mechanical analyses. (a,b) Stress relaxation test results. Stress ratio of the (a) PEA elastomer and (b) DN elastomer as a function of relaxation time. (c,d) Cycle tensile test results of the (c) PEA elastomer and (d) DN elastomer performed at a strain rate $0.14 \mathrm{~s}^{-}$
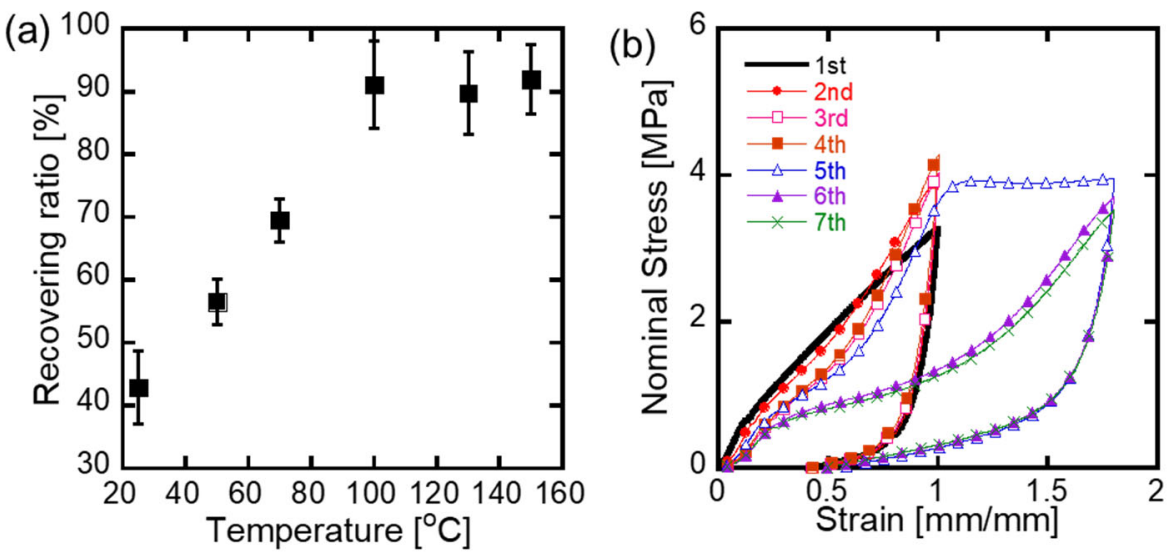

Figure 6. Recoverability of the DN elastomer. (a) Recovering ratio of the DN elastomer as a function of the annealing temperature. (b) Cycle loading-unloading curves of the DN elastomer. The sample was annealed at $130{ }^{\circ} \mathrm{C}$ for $5 \mathrm{~min}$ between each cycle. 


\section{Discussion}

Based on these results, the synergetic toughening mechanism of the cellulose/PEA DN elastomer is described. The previous works about sacrificial bond principle tell us that the toughening of the cellulose/PEA DN elastomer is the result of the damage zone formation around a crack tip, where the brittle first network working as s-bonds ruptures internally and dissipates much energy [7]. Such preferential internal rupture in the cellulose/PEA DN elastomer was observed as a large mechanical hysteresis in the cycle tensile test. With consideration of the molecular independency of the two networks suggested by the X-ray diffraction and the FT-IR spectra, this internal rupture of the DN elastomer should correspond to the rupture of either the H-bonds or the covalent bonds within the cellulose first network. As suggested by the cycle tensile test, the observed mechanical hysteresis of the DN elastomer was essentially reversible. Moreover, although the cellulose network should have chemical cross-linking, the rupture of covalent bonds in the stretched cellulose/PEA DN elastomer was not detected experimentally (Figure S2). Therefore, it was expected that the Hbonds with reversible nature mainly work as s-bonds in the cellulose/PEA DN elastomer, which has been observed as the fast relaxation component $(\tau \sim 1 \mathrm{~s})$ with the stress relaxation test. Figure 7 shows the estimated schematic H-bond rupture process of the cellulose/PEA DN elastomer. The cellulose first network structure is maintained mainly by the H-bonds. At low strain, as the amount of ruptured H-bonds remains small, the original cellulose network can be recovered by re-formation of the ruptured H-bonds by thermal annealing. On the other hand, when strain reaches a certain (yield) point, rupture of a large number of the H-bonds induces plastic flow of the cellulose network. Thus, after reaching this yield point, the deformed cellulose network no longer recovers its original structure even after annealing. At any strain, the second PEA network functions as the stretchable matrix to maintain the integrity of the elastomer. 
From the viewpoint of mechanical response at each strain, the cellulose/PEA DN elastomer can be considered as an "ideal" elastomer following the s-bond principle. During practical use, an elastomer is regularly subjected to small deformations. At low strain, the rupture of the sacrificial hydrogen bonds in the DN elastomer can be recovered by annealing. This means that during regular use, the cellulose/PEA DN elastomer can be considered as a tough and non-deteriorated elastomer. In contrast, at a higher strain that is not usually experienced in regular use, extremely large energy is dissipated by the irreversible rupture of the sacrificial hydrogen bonds, which sufficiently increases the toughness of the DN elastomer. The role of such an irreversible structure change at high strain can be compared to that of a fuse for an electric circuit. If a large force is accidentally applied to the DN elastomer, strong and irreversible hydrogen bonds in the cellulose network suffer sacrificial rupture that prevents the global failure of the DN elastomer. This fuse-like toughening mechanism results in a high reliability against an unexpected force applied to the cellulose/PEA DN elastomers.

Finally, we make some comments on the difference between the filled elastomers and the cellulose/PEA DN elastomers. Conventional filled elastomers are toughened by addition of stiff and strong materials like CBs. Although their toughening mechanism are not completely understood, debonding of the interfacial Van der Waals interactions between the fillers and the rubbery matrix during the deformation, which dissipates large energy, strongly contributes to high toughness of the filled elastomers. Since the energy dissipation occurs only at the interface (not inside) of fillers, massive fillers are required for the effective energy dissipation and toughening. On the other hand, tough soft materials based on the s-bond principle like the cellulose/PEA DN elastomers are toughened by rupture of the s-bonds during the deformation. The rupture of the s-bonds efficiently occurs inside the materials during the deformation. This is one of the reasons why a few $\mathrm{wt} \%$ of the cellulose network effectively 
toughens the PEA elastomer unlike the filled elastomers which require more than $10 \mathrm{vol} \%$ of fillers for the effective toughening.

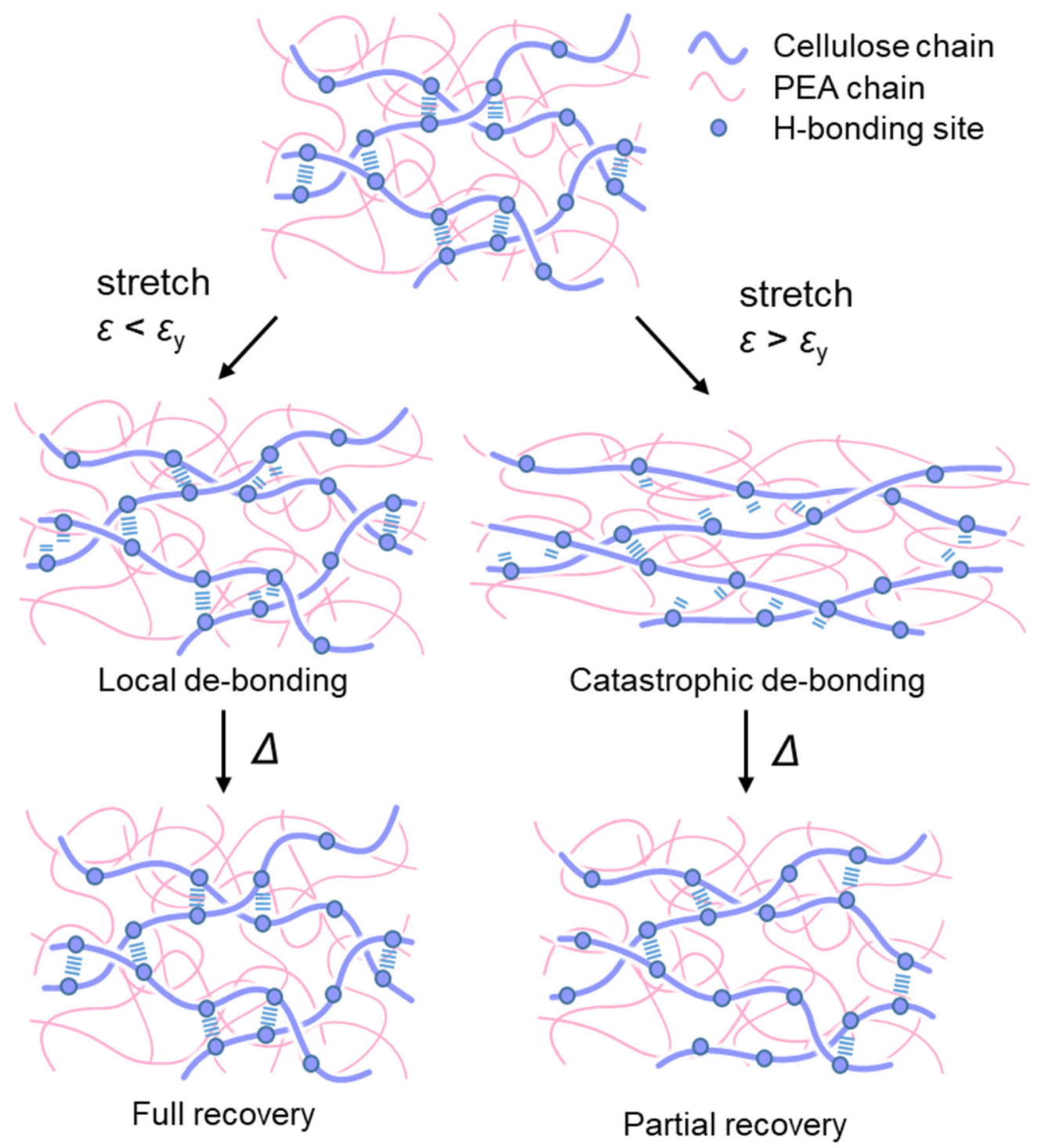

Figure 7. Schematic illustrations of the proposed deformation mechanism of the cellulose/PEA DN elastomer. Stretching of the sample induces rupture of the hydrogen $(\mathrm{H})$ bonds between the cellulose chains. Before reaching the yield point, small deformation induces localized H-bond breaking, which can be healed by thermal annealing. Once the yield point is reached, catastrophic H-bond breaking induces plastic flow of the cellulose network, which leads to irreversible deformation of the DN elastomer even after annealing. 
In conclusion, a strong, tough, and durable cellulose/PEA DN elastomer was successfully fabricated based on the sacrificial bond principle. Addition of only $2.55 \mathrm{wt} \%$ of the cellulose network as a brittle component sufficiently improved the stiffness, strength, and toughness of the PEA elastomer while maintaining its transparency. At low strain, the hydrogen bonds in the cellulose network showed reversible energy dissipation to bear daily stress. At high strain, which is rarely applied to the material, the cellulose network showed irreversible but large energy dissipation, which emergently prevented crack propagation. This shows that the cellulose/PEA DN elastomer is a good candidate among the eco-friendly and reliable elastomers that are preferred for various industrial applications such as the manufacture of sporting goods and tires.

\section{Acknowledgements}

This research was partially funded by the ImPACT Program of the Council for Science, Technology and Innovation (Cabinet Office, Government of Japan). This work was also partially supported by JSPS KAKENHI (Grant no. JP17H06144, JP17H04891, JP26870008, and 17H06376). The Institute for Chemical Reaction Design and Discovery (ICReDD) was established by the World Premier International Research Initiative (WPI), MEXT, Japan. Synchrotron radiation experiments were performed at the BL40B2 of SPring-8 with the approval of the Japan Synchrotron Radiation Research Institute (JASRI) (Proposal No. 2015B1140). J. M. thanks Dr. Yoshinori Katsuyama, Ms. Yuki Maida, Ms. Yukiko Hane (Hokkaido University) for their help with the experiments. The authors acknowledge Dr. Daniel R. King (Hokkaido University) for constructive discussions.

\section{References}

[1] J.T. Byers, Ch. 3 Fillers Part I Carbon Black, in: M. Morton (Ed.) Rubber Technology third Ed., Springer-Verlag, Berlin-Heidelberg, 1999, pp.59-85. 
[2] K. Ito, Novel Cross-Linking Concept of Polymer Network: Synthesis, Structure, and Properties of Slide-Ring Gels with Freely Movable Junctions, Polym. J. 39 (2007) 489-499. https://doi.org/10.1295/polymj.PJ2006239.

[3] A.B. Imran, K. Esaki, H. Gotoh, T. Seki, K. Ito, Y. Sakai, Y Takeoka, Extremely stretchable thermosensitive hydrogels by introducing slide-ring polyrotaxane cross-linkers and ionic groups into the polymer network, Nat. Commun. 5 (2011) 5124. https://doi.org/10.1038/ncomms6124.

[4] K. Haraguchi, Synthesis and properties of soft nanocomposite materials with novel organic/inorganic network structures, Polym. J. 43 (2011) 223-241. https://doi.org/10.1038/pj.2010.141.

[5] Q. Wang, J.L. Mynar, M. Yoshida, E. Lee, M. Lee, K. Okuro, K. Kinbara, T. Aida, High-water-content mouldable hydrogels by mixing clay and a dendritic molecular binder, Nature 463 (2010) 339-343. https://doi.org/10.1038/nature08693.

[6] J.P. Gong, Why are double network hydrogels so tough?, Soft Matter 6 (2010) 25832590. https://doi.org/10.1039/B924290B.

[7] T. Nakajima, Generalization of the sacrificial bond principle for gel and elastomer toughening, Polym. J. 49 (2017) 477-485. https://doi.org/10.1038/pj.2017.12.

[8] J.P. Gong, Y. Katsuyama, T. Kurokawa, Y. Osada, Double-Network Hydrogels with Extremely High Mechanical Strength, Adv. Mater. 15 (2003) 1155-1158. https://doi.org/10.1002/adma.200304907.

[9] Y.-H. Na, Y. Tanaka, Y. Kawauchi, H. Furukawa, T. Sumiyoshi, J.P. Gong, Y. Osada, Necking Phenomenon of Double-Network Gels, Macromolecules 39 (2006) 4641-4645. https://doi.org/10.1021/ma060568d.

[10] R.E. Webber, C. Creton, H.R. Brown, J.P. Gong, Large Strain Hysteresis and Mullins Effect of Tough Double-Network Hydrogels, Macromolecules 40 (2007) 2919-2927. https://doi.org/10.1021/ma062924y. 
[11] Y. Tanaka, Y. Kawauchi, T. Kurokawa, H. Furukawa, T. Okajima, J.P. Gong, Localized Yielding Around Crack Tips of Double-Network Gels, Macromol. Rapid Commun. 29 (2008) 1514-1520. https://doi.org/10.1002/marc.200800227.

[12] Q.M. Yu, Y. Tanaka, H. Furukawa, T. Kurokawa, J.P. Gong, Direct Observation of Damage Zone around Crack Tips in Double-Network Gels, Macromolecules 42 (2009) 38523855. https://doi.org/10.1021/ma900622s.

[13] Y. Tanaka, R. Kuwabara, Y.-H. Na, T. Kurokawa, J.P. Gong, Y. Osada, Determination of Fracture Energy of High Strength Double Network Hydrogels, J. Phys. Chem. B 109 (2005) 11559-11562. https://doi.org/10.1021/jp0500790.

[14] T. Nakajima, H. Furukawa, Y. Tanaka, T. Kurokawa, Y. Osada, J.P. Gong, True Chemical Structure of Double Network Hydrogels, Macromolecules 42 (2009) 2184-2189. https://doi.org/10.1021/ma802148p.

[15] M.A. Haque, T. Kurokawa, G. Kamita, J.P. Gong, Lamellar Bilayers as Reversible Sacrificial Bonds to Toughen Hydrogel: Hysteresis, Self-recovery, Fatigue Resistance, and Crack Blunting, Macromolecules 44 (2011) 8916-8924. https://doi.org/10.1021/ma201653t. [16] J.-Y. Sun, X. Zhao, W.R.K. Illeperuma, O. Chaudhuri, K. H. Oh, D. J. Mooney, J. J. Vlassak, Z. Suo, Highly stretchable and tough hydrogels, Nature 489 (2012) 133-136. https://doi.org/10.1038/nature11409.

[17] T.L. Sun, T. Kurokawa, S. Kuroda, A.B. Ihsan, T. Akasaki, K. Sato, M.A. Haque, T. Nakajima, J.P. Gong, Physical Hydrogels Composed of Polyampholytes Demonstrate High Toughness and Viscoelasticity, Nat. Mater. 12 (2013) 932-937. https://doi.org/10.1038/nmat3713.

[18] K. Sato, T. Nakajima, T. Hisamatsu, T. Nonoyama, T. Kurokawa, J.P. Gong, Phase Separation-Induced Anomalous Stiffening, Toughening, and Self-healing of Polyacrylamide Gels, Adv. Mater. 27 (2015) 6990-6998. https://doi.org/10.1002/adma.201502967. 
[19] H.J. Zhang, T.L. Sun, A.K. Zhang, Y. Ikura, T. Nakajima, T. Nonoyama, T.

Kurokawa, O. Ito, H. Ishitobi, J.P. Gong, Tough Physical Double-Network Hydrogels Based on Amphiphilic Triblock Copolymers, Adv. Mater. 28 (2016) 4884-4890.

https://doi.org/10.1002/adma.201600466.

[20] E. Ducrot, Y. Chen, M. Bulters, R.P. Sijbesma, C. Creton, Toughening elastomers with sacrificial bonds and watching them break, Science 344 (2014) 186-189. https://doi.org/10.1126/science.1248494.

[21] E. Ducrot, H. Montes, C. Creton, Macromolecules 48 (2015) 7945-7952. https://doi.org/10.1021/acs.macromol.5b01979.

[22] S.J. Eichhorn, A. Dufresne, M. Aranguren, N.E. Marcovich, J.R. Capadona, S. J. Rowan, C. Weder, W. Thielemans, M. Roman, S. Renneckar, W. Gindl, S. Veigel, J. Keckes, H. Yano, K. Abe, M. Nogi, A. N. Nakagaito, A. Mangalam, J. Simonsen, A.S. Benight, A. Bismarck, L.A. Berglund, T. Peijs, Review: current international research into cellulose nanofibres and nanocomposites, J. Mater. Sci. 45 (2010) 1-33. https://doi.org/10.1007/s10853-009-3874-0.

[23] H. Yano, J. Sugiyama, A. N. Nakagaito, M. Nogi, T. Matsuura, M. Hikita, K. Handa, Optically Transparent Composites Reinforced with Networks of Bacterial Nanofibers, $A d v$. Mater. 17 (2005) 153-155. https://doi.org/10.1002/adma.200400597.

[24] H. Fukuzumi, T. Saito, T. Iwata, Y. Kumamoto, A. Isogai, Transparent and High Gas Barrier Films of Cellulose Nanofibers Prepared by TEMPO-Mediated Oxidation, Biomacromolecules 10 (2009) 162-165. https://doi.org/10.1021/bm801065u.

[25] A. Pei, J. M. Malho, J. Ruokolainen, Q. Zhou, L.A. Berglund, Strong Nanocomposite Reinforcement Effects in Polyurethane Elastomer with Low Volume Fraction of Cellulose Nanocrystals, Macromolecules 44 (2011) 4422-4427. https://doi.org/10.1021/ma200318k.

[26] G. Siqueira, S.T.-Lingua, J. Bras, D.D.S. Perez, A. Dufresnel, Mechanical properties of natural rubber nanocomposites reinforced with cellulosic nanoparticles obtained from 
combined mechanical shearing, and enzymatic and acid hydrolysis of sisal fibers, Cellulose 18 (2011) 57-65. https://doi.org/10.1007/s10570-010-9463-1.

[27] W.P.F. Neto, M. Mariano, I. Souza, V. Silva, H. A. Silvério, J.-L. Putaux, H. Otaguro, D. Pasquini, A. Dufresne, Mechanical properties of natural rubber nanocomposites reinforced with high aspect ratio cellulose nanocrystals isolated from soy hulls, Carbohydr. Polym. 153 (2016) 143-152. https://doi.org/10.1016/j.carbpol.2016.07.073.

[28] A. Nakayama, A. Kakugo, J. P. Gong, Y. Osada, M. Takai, T. Erata, S. Kawano, High Mechanical Strength Double-Network Hydrogel with Bacterial Cellulose, Adv. Funct. Mater. 14 (2014) 1124-1128. https://doi.org/10.1002/adfm.200305197.

[29] Y. Hagiwara, A. Putra, A. Kakugo, H. Furukawa, J.P. Gong, Ligament-like Tough Double Network Hydrogel Based on Bacterial Cellulose, Cellulose 17 (2010) 93-101. https://doi.org/10.1007/s10570-009-9357-2.

[30] C.L. McCormick, P.A. Callais, B. H. Hutchinson, Solution studies of cellulose in lithium chloride and N,N-dimethylacetamide, Macromolecules 18 (1985) 2394-2401. https://doi.org/10.1021/ma00154a010.

[31] D. Ishii, D. Tatsumi, T. Matsumoto, Effect of Solvent Exchange on the Solid Structure and Dissolution Behavior of Cellulose, Biomacromolecules 4 (2003) 1238-1243. https://doi.org/10.1021/bm034065g.

[32] H. Kono, S. Fujita, Biodegradable superabsorbent hydrogels derived from cellulose by esterification crosslinking with 1,2,3,4-butanetetracarboxylic dianhydride, Carbohydr. Polym. 87 (2012) 2582-2588. https://doi.org/10.1016/j.carbpol.2011.11.045.

[33] Y. Maréchal, H. Chanzy, The hydrogen bond network in I $\beta$ cellulose as observed by infrared spectrometry, J. Mol. Struct. 523 (2000) 183-196. https://doi.org/10.1016/S00222860(99)00389-0. 
[34] K. Tsunoda, J.J.C. Busfield, C.K.L. Davies, A.G. Thomas, Effect of materials variables on the tear behaviour of a non-crystallising elastomer, J. Mater. Sci. 35 (2000) 5187-5198. https://doi.org/10.1023/A:1004860522186.

[35] H.W. Greensmith, Rupture of rubber. IV. Tear properties of vulcanizates containing carbon black, J. Polym. Sci. 21 (1956) 175-187. https://doi.org/10.1002/pol.1956.120219802 [36] Y. Morishita, K. Tsunoda, K. Urayama, Velocity transition in the crack growth dynamics of filled elastomers: Contributions of nonlinear viscoelasticity, Phys. Rev. E 93 (2016) 043001. https://doi.org/10.1103/PhysRevE.93.043001.

[37] A. Kubo, Y. Umeno, Velocity mode transition of dynamic crack propagation in hyperviscoelastic materials: A continuum model study, Sci. Rep. 7 (2017) 42305. https://doi.org/10.1038/srep42305.

[38] N. Sakumichi, K. Okumura, Exactly solvable model for a velocity jump observed in crack propagation in viscoelastic solids, Sci. Rep. 7 (2017) 8065. https://doi.org/10.1038/s41598-017-07214-8.

[39] S. Ahmed, T. Nakajima, T. Kurokawa, M.A. Haque, J.P. Gong, Brittle-ductile transition of double network hydrogels: Mechanical balance of two networks as the key factor, Polymer 55 (2014) 914-923. https://doi.org/10.1016/j.polymer.2013.12.066.

[40] T. Matsuda, T. Nakajima, Y. Fukuda, W. Hong, T. Sakai, T. Kurokawa, U.-I. Chung, J.P. Gong, Macromolecules 49 (2016) 1865-1872. https://doi.org/10.1021/acs.macromol.5b02592. 


\section{WILEY-VCH}

Supplementary Information

Tough Double Network Elastomers Reinforced by the Amorphous Cellulose Network

Joji Murai, Tasuku Nakajima*, Takahiro Matsuda, Katsuhiro Tsunoda, Takayuki Nonoyama, Takayuki Kurokawa, Jian Ping Gong*

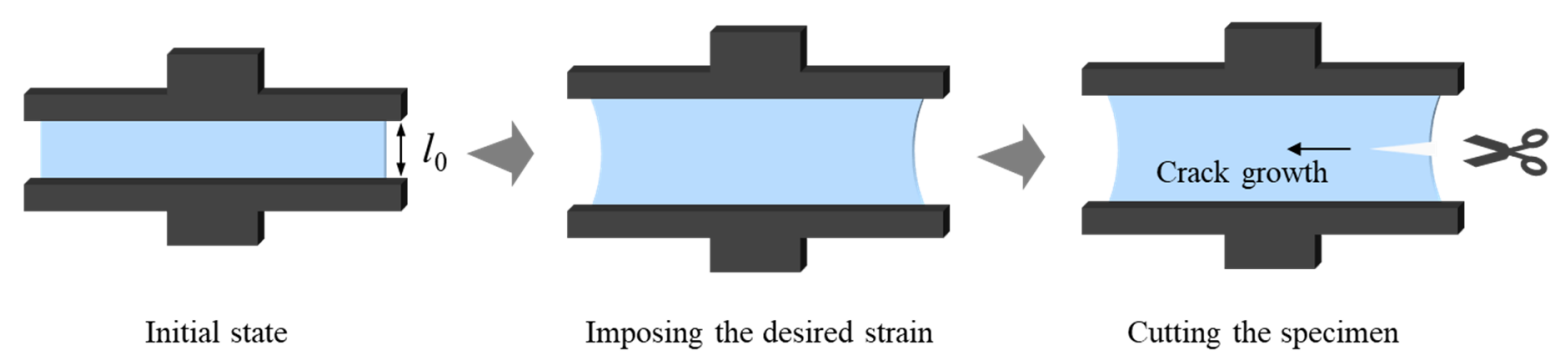

Figure S1: Experimental procedure for the crack growth test. 

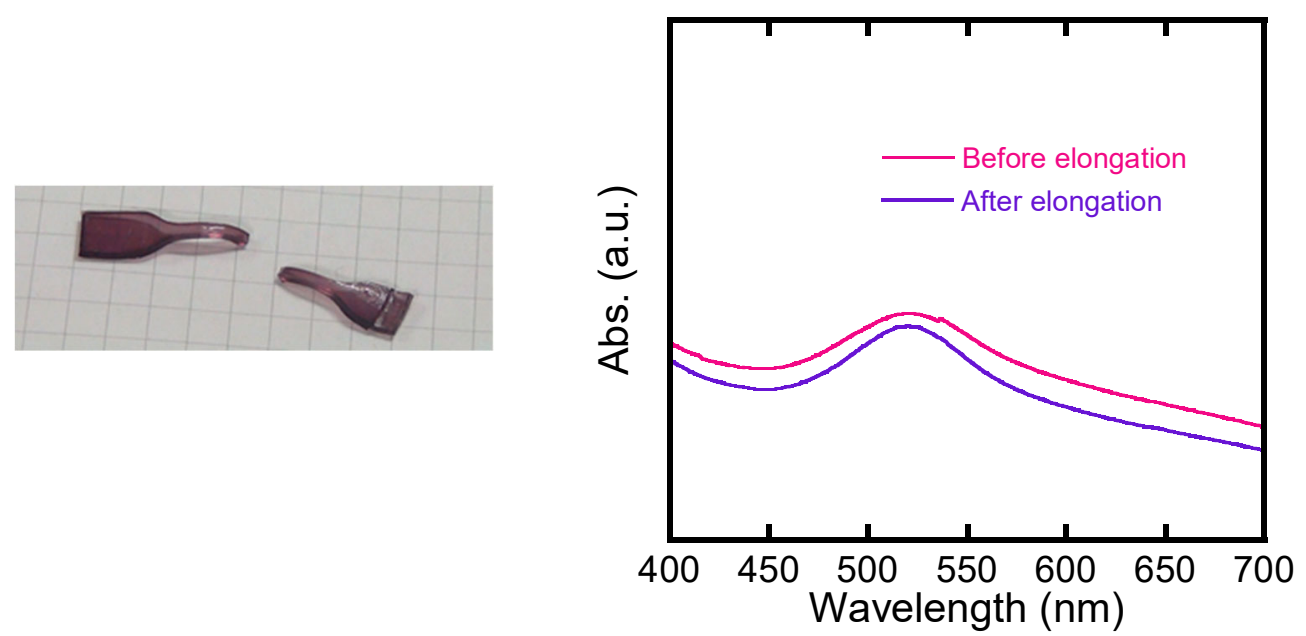

Figure S2: Radical detection in the stretched elastomers. 2,2-diphenyl-1-picrylhydrazyl (DPPH) was used as the radical detection reagent. DPPH originally has purple color, but it is breached when exposed to radical (H. T. Baytekin et al., Angew. Chem., 2012, 51, 3596.). The cellulose/PEA DN elastomers was immersed in excess dichloromethane containing $300 \mu \mathrm{M}$ of DPPH and then dried for $4 \mathrm{~h}$. The sample containing DPPH was cut into the dumbbell shape standardized as per the JIS-K6251-7 size and then uniaxially elongated at constant velocity of $100 \mathrm{~mm} / \mathrm{min}$ until their fracture. The graph shows the transmission visible light spectrum of the DN elastomer near the fracture interface (curves are offset for clarification). No obvious color and spectrum change were observed, suggesting no significant radical generation (=covalent bond fracture) was observed near fracture interface of the cellulose/PEA DN elastomer. 